\title{
The Synchronization Power of Coalesced Memory Accesses
}

\author{
Phuong Hoai Ha ${ }^{1}$, Philippas Tsigas ${ }^{2}$, and Otto J. Anshus ${ }^{1}$ \\ 1 University of Troms $\varnothing$, Department of Computer Science, Faculty of Science, \\ NO-9037 Troms $\varnothing$, Norway, \\ \{phuong, otto\}@cs.uit.no \\ 2 Chalmers University of Technology, Department of Computer Science and \\ Engineering, SE-412 96 Göteborg, Sweden, \\ tsigas@chalmers.se
}

\begin{abstract}
Multicore processor architectures have established themselves as the new generation of processor architectures. As part of the one core to many cores evolution, memory access mechanisms have advanced rapidly. Several new memory access mechanisms have been implemented in many modern commodity multicore processors. Memory access mechanisms, by devising how processing cores access the shared memory, directly influence the synchronization capabilities of the multicore processors. Therefore, it is crucial to investigate the synchronization power of these new memory access mechanisms.

This paper investigates the synchronization power of coalesced memory accesses, a family of memory access mechanisms introduced in recent large multicore architectures like the CUDA graphics processors. We first design three memory access models to capture the fundamental features of the new memory access mechanisms. Subsequently, we prove the exact synchronization power of these models in terms of their consensus numbers. These tight results show that the coalesced memory access mechanisms can facilitate strong synchronization between the threads of multicore processors, without the need of synchronization primitives other than reads and writes. In the case of the contemporary CUDA processors, our results imply that the coalesced memory access mechanisms have consensus numbers up to sixteen.
\end{abstract}

\section{Introduction}

One of the fastest evolving multicore architectures is the graphics processor one. The computational power of graphics processors (GPUs) doubles every ten months, surpassing the Moore's Law for traditional microprocessors [13]. Unlike previous GPU architectures, which are single-instruction multiple-data (SIMD), recent GPU architectures (e.g. Compute Unified Device Architecture (CUDA) [2]) are single-program multiple-data (SPMD). The latter consists of multiple SIMD multiprocessors of which each, at the same time, can execute a different instruction. This extends the set of applications on GPUs, which are no longer restricted to follow the SIMD-programming model. Consequently, GPUs are 
emerging as powerful computational co-processors for general-purpose computations.

Along with their advances in computational power, GPUs memory access mechanisms have also evolved rapidly. Several new memory access mechanisms have been implemented in current commodity graphics/media processors like the Compute Unified Device Architecture (CUDA) [2] and Cell BE architecture [1]. For instance, in CUDA, single-word write instructions can write to words of different size and their size (in bytes) is no longer restricted to be a power of two [2]. Another advanced memory access mechanism implemented in CUDA is the coalesced global memory access mechanism. The simultaneous global memory accesses by each thread of a SIMD multiprocessor, during the execution of a single read/write instruction, are coalesced into a single aligned memory access if the simultaneous accesses follow the coalescence constraint [2]. The access coalescence takes place even if some of the threads do not actually access memory. It is well-known that memory access mechanisms, by devising how processing cores access the shared memory, directly influence the synchronization capabilities of multicore processors. Therefore, it is crucial to investigate the synchronization power of the new memory access mechanisms.

Research on the synchronization power of memory access operations (or objects) in conventional architectures has received a great amount of attention in the literature. The synchronization power of memory access objects/mechanisms is conventionally determined by their consensus-solving ability, namely their consensus number [10]. The consensus number of an object type is either the maximum number of processes for which the consensus problem can be solved using only objects of this type and registers, or infinity if such a maximum does not exist. For hard real-time systems, it has been shown that any object with consensus number $n$ is universal ${ }^{3}$ for any numbers of processes running on $n$ processors [14]. For systems that allow processes to simultaneously access $m$ objects of type $T$ in one atomic operation (or multi-object operation), upper and lower bounds on the consensus number of the multi-object called type $T^{m}$ have been provided for the base type $T$ with consensus number greater than or equal to two $[4,11$, $16]$. In the case of registers (which have consensus number one), the $m$-register assignment, which allows processes to write to $m$ arbitrary registers atomically, has been proven to have consensus number $(2 m-2)$, for $m>1$ [10].

Note that the aforementioned CUDA coalesced memory accesses are neither the atomic $m$-register assignment [10] nor the multi-object types $[4,11,16]$. They are not the atomic $m$-register assignment since they do not allow processes to atomically write to $m$ arbitrary memory words; instead, processes can atomically write to $m$ memory words only if the $m$ memory words are located within an aligned size-bounded memory portion (i.e. memory alignment restriction) (cf. Section 2). The CUDA coalesced memory accesses are not the multi-object type since their base object type $T$ is the conventional memory word, which has consensus number less than two.

\footnotetext{
${ }^{3}$ An object is universal in a system of $n$ processes iff it has a consensus number not
} lower than $n$. 
This paper investigates the consensus number of the new memory access mechanisms implemented in current graphics processor architectures. We first design three new memory access models to capture the fundamental features of the new memory access mechanisms. Subsequently we prove the exact synchronization power of these models in terms of their respective consensus number. These tight results show that the new memory access mechanisms can facilitate strong synchronization between the threads of multicore processors, without the need of synchronization primitives other than reads and writes.

We first design a new memory access model, the svword model where svword stands for the size-varying word access, the first of the two aforementioned advanced memory access mechanisms implemented in CUDA. Unlike single-word assignments in conventional processor architectures, the new single-word assignments can write to words of size $b$ (in bytes), where $b$ can vary from 1 to an upper bound $B$ and $b$ is no longer restricted to be a power of 2 (e.g. type float3 in [2]). By carefully choosing $b$ for the single-word assignments, we can partly overlap the bytes written by two assignments, namely each of the two assignments has some byte(s) that is not overwritten by the other overlapping assignment (cf. Figure 1(a) for an illustration). Note that words of different size must be aligned from the address base of the memory. This memory alignment constraint prevents single-word assignments in conventional architectures from partly overlapping each other since the word-size is restricted to be a power of two. On the other hand, since the new single-word assignment can write to a subset of bytes of a big word (e.g. up to 16 bytes) and leave the other bytes of the word intact, the size of values to be written becomes a significant factor. The assignment can atomically write $B$ values of size 1 (instead of just one value of size $B$ ) to $B$ consecutive memory locations. The observation has motivated us to develop the svword model.

Inspired by the coalesced memory accesses, the second of the aforementioned advanced memory access mechanisms, we design two other models, the aiword and asvword models, to capture the fundamental features of the mechanism. The mechanism coalesces simultaneous read/write instructions by each thread of a SIMD multiprocessor into a single aligned memory access even if some of the threads do not actually access memory [2]. This allows each SIMD multiprocessor (or process) to atomically write to an arbitrary subset of the aligned memory units that can be written by a single coalesced memory access. We generally model this mechanism as an aligned-inconsecutive-word access, aiword, in which the memory is aligned to $A$-unit words and a single-word assignment can write to an arbitrary non-empty subset of the $A$ units of a word. Note that the single-aiword assignment is not the atomic $m$-register assignment [10] due to the memory alignment restriction ${ }^{4}$. Our third model, asvword, is an extension of the second model aiword in which aiword's $A$ memory units are now replaced by $A$ svwords of the same size $b$. This model is inspired by the fact that the read/write

\footnotetext{
${ }^{4}$ In this paper, we use term "single" in single- word assignment when we want to emphasize that the assignment is not the multiple assignment [10].
} 
instructions of different coalesced global memory accesses can access words of different size [2].

The contributions of this paper can be summarized as follows:

- We develop a general memory access model, the svword model, to capture the fundamental features of the size-varying word accesses. In this model, a single-word assignment can write to a word comprised of $b$ consecutive memory units, where $b$ can be any integer between 1 and an upper bound $B$. We prove that the single-svword assignment has consensus number $3, \forall B \geq 5$, and that consensus number 3 is also the upper bound of consensus numbers of the single-svword assignment $\forall B \geq 2$. We also introduce a technique to minimize the size of (proposal) values in consensus algorithms, which allows a single-word assignment to write many values atomically and handle the consensus problem for several processes (cf. Section 3).

- We develop a general memory access model, the aiword model, to capture the fundamental features of the coalesced memory accesses. The second model is an aligned-inconsecutive-word access model in which the memory is aligned to $A$-unit words and a single-word assignment can write to an arbitrary non-empty subset of the $A$ units of a word. We present a wait-free consensus algorithm for $N=\left\lfloor\frac{A+1}{2}\right\rfloor$ processes using only single-aiword assignments and subsequently prove that the single-aiword assignment has consensus number exactly $N=\left\lfloor\frac{A+1}{2}\right\rfloor$ (cf. Section 4 ).

- We develop a general memory access model, asvword, to capture the fundamental features of the combination of the size-varying word accesses and the coalesced memory accesses. The third model is an extension of the second model aiword in which aiword's $A$ units are $A$ svwords of the same size $b, b \in\{1, B\}$ (cf. Section 5). We prove that the consensus number of the single-asvword assignment is exactly $N$, where

$$
N= \begin{cases}\frac{A B}{2}, & \text { if } A=2 t B, t \in \mathbb{N}^{*} \text { (positive integers) } \\ \frac{(A-B) B}{2}+1, & \text { if } A=(2 t+1) B, t \in \mathbb{N}^{*} \\ \left\lfloor\frac{A+1}{2}\right\rfloor, & \text { if } B=t A, t \in \mathbb{N}^{*}\end{cases}
$$

In the case of the contemporary CUDA processors (with compute capability up to 1.1) in which $A=16$ and $B=2$, the consensus number of the asvword model is sixteen.

The rest of this paper is organized as follows. Section 2 presents the three new memory access models. Sections 3, 4 and 5 present the exact consensus numbers of the first, second and third models, respectively.

Due to space limitations, we present here only intuitions behind the consensus number results. Complete proofs of the results can be found in the full version of this paper [9]. 


\section{Models}

Before describing the details of each of the three new memory access models, we present the common properties of all these three models. The shared memory in the three new models is sequentially consistent $[3,12]$, which is weaker than the linearizable one [5] assumed in most of the previous research on the synchronization power of the conventional memory access models [10]. Processes are asynchronous. The new models use the conventional 1-dimensional memory address space. In these models, one memory unit is a minimum number of consecutive bytes/bits which a basic read/write operation can atomically read from/write to (without overwriting other unintended bytes/bits). These memory models address individual memory units. Memory is organized so that a group of $n$ consecutive memory units called word can be stored or retrieved in a single basic write or read operation, respectively, and $n$ is called word size. Words of size $n$ must always start at addresses that are multiples of $n$, which is called alignment restriction as defined in the conventional computer architecture.

The first model is a size-varying-word access model (svword) in which a single $\mathrm{read} /$ write operation can atomically read from/write to a word consisting of $b$ consecutive memory units, where $b$ can be any integer between 1 and an upper bound $B$ and is called svword size. The upper bound $B$ is the maximum number of consecutive units which a basic read/write operation can atomically read from/write to. Svwords of size $b$ must always start at addresses that are multiples of $b$ due to the memory alignment restriction. We denote $b$-svword to be an svword consisting of $b$ units, $b$-svwrite to be a $b$-svword assignment and $b$-svread to be a $b$-svword read operation. Reading a unit $U$ is denoted by $1-\operatorname{svread}(U)$ or just by $U$ for short. This model is inspired by the CUDA graphics processor architecture in which basic read/write operations can atomically read from/write to words of different size (cf. types float1, float2, float3 and float4 in [2], Section 4.3.1.1). Figure 1(a) illustrates how 2-svwrite, 3-svwrite and 5-svwrite can partly overlap their units with addresses from 14 to 20 , with respect to the memory alignment restriction.

The second model is an aligned-inconsecutive-word access model (aiword) in which the memory is aligned to $A$-unit words and a single read/write operation can atomically read from/write to an arbitrary non-empty subset of the $A$ units of a word, where $A$ is a constant. Aiwords must always start at addresses that are multiples of $A$ due to the memory alignment restriction. We denote $A$-aiword to be an aiword consisting of $A$ units, A-aiwrite to be an $A$-aiword assignment and $A$-airead to be an $A$-aiword read operation. Reading only one unit $U$ (using airead) is denoted by $U$ for short. In the aiword model, an aiwrite operation executed by a process cannot atomically write to units located in different aiwords due to the memory alignment restriction.

Figure 1(b) illustrates the aiword model with $A=8$ in which the aiword consists of eight consecutive units with addresses from 8 to 15 . Unlike in the svword model, the assignment in the aiword model can atomically write to inconsecutive 


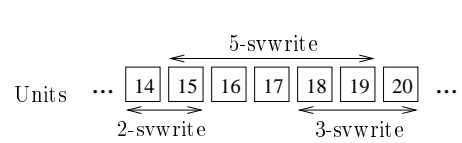

(a) The first model svword

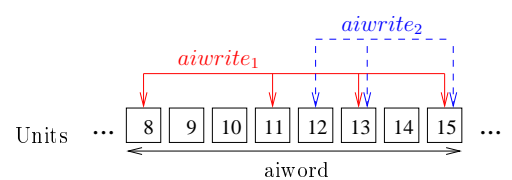

(b) The second model aiword

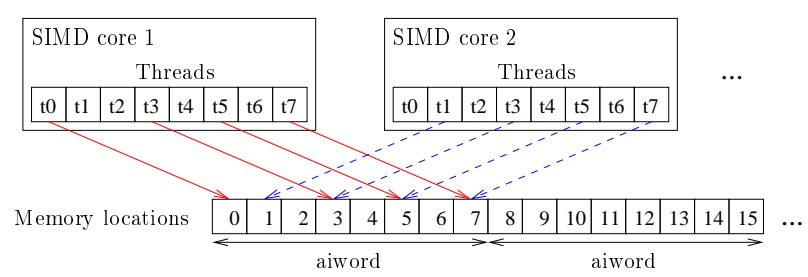

(c) The coalesced memory access

Fig. 1. Illustrations for the first model, size-varying-word access (svword), the second model, aligned-inconsecutive-word access (aiword) and the coalesced memory access.

units of the eight units: aiwrite $_{1}$ atomically writes to four units 8,11,13 and 15; aiwrite $_{2}$ writes to three units 12,13 and 15 .

This model is inspired by the coalesced global memory accesses in the CUDA architecture [2]. The CUDA architecture can be generalized to an abstract model of a MIMD ${ }^{5}$ chip with multiple SIMD cores sharing memory. Each core can process $A$ threads simultaneously in a SIMD manner, but different cores can simultaneously execute different instructions. The instance of a program that is being sequentially executed by one SIMD core is called process. Namely, each process consists of $A$ parallel threads that are running in SIMD manner. The process accesses the shared memory using the CUDA memory access models. In CUDA, the simultaneous global memory accesses by each thread of a SIMD core during the execution of a single read/write instruction can be coalesced into a single aligned memory access. The coalescence happens even if some of the threads do not actually access memory (cf. [2], Figure 5-1). This allows a SIMD core (or a process consisting of $A$ parallel threads running in a SIMD manner) to atomically access multiple memory locations that are not at consecutive addresses.

Figure 1(c) illustrates the coalesced memory access, where $A=8$. The left SIMD core can write atomically to four memory locations $0,3,5$ and 7 by letting only four of its eight threads, $t 0, t 3, t 5$ and $t 7$, simultaneously execute a write operation (i.e. divergent threads). The right SIMD core can write atomically to its own memory location 1 and shared memory locations 3,5 and 7 by letting only four threads $t 1, t 3, t 5$ and $t 7$ simultaneously execute a write operation. Note that

\footnotetext{
${ }^{5}$ MIMD: Multiple-Instruction-Multiple-Data
} 


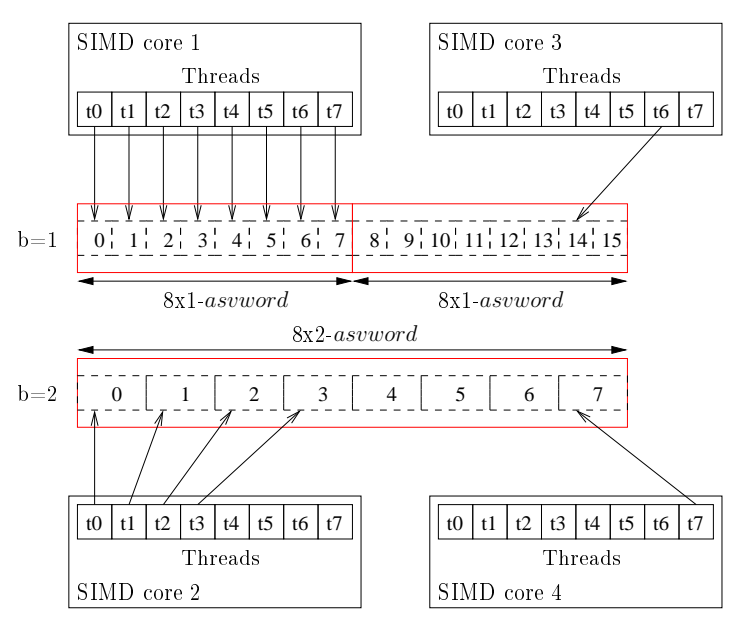

Fig. 2. An illustration for the asvword model.

the CUDA architecture allows threads from different SIMD cores to communicate through the global shared memory [7].

The third model is a coalesced memory access model (asvword), an extension of the second model aiword in which aiword's $A$ units are now replaced by $A$ svwords of the same size $b, b \in[1, B]$. Namely, the second model aiword is a special case of the third model asvword where $B=1$. This model is inspired by the fact that in CUDA the read/write instructions of different coalesced global memory accesses can access words of different size. Let Axb-asvword be the asvword that is composed of $A$ svwords of which each consists of $b$ memory units. Axb-asvwords whose size is $A \cdot b$ must always start at addresses that are multiples of $A \cdot b$ due to the memory alignment restriction. We denote $A \mathrm{x} b$-asvwrite to be an $A \mathrm{x} b$-asvword assignment and $A \mathrm{x} b$-asvread to be an $A \mathrm{x} b$-asvword read operation. Reading only one unit $U$ (using Ax1-asvread) is denoted by $U$ for short. Due to the memory alignment restriction, an $A \mathrm{x} b$-asvwrite operation cannot atomically write to $b$ svwords located in different $A \mathrm{x} b$-asvwords. Since in reality $A$ and $B$ are a power of 2 , in this model we assume that either $B=k \cdot A, k \in \mathbb{N}^{*}$ (in the case of $B \geq A$ ) or $A=k \cdot B, k \in \mathbb{N}^{*}$ (in the case of $B<A$ ). (At the moment, CUDA supports the atomic coalesced memory access to only words of size 4 and 8 bytes (i.e. only svwords consisting of 1 and 2 units in our definition), cf. Section 5.1.2.1 in [2]). For the sake of simplicity, we assume that $b \in\{1, B\}$ holds. A more general model with $b=2^{c}, c=0,1, \cdots, \log _{2} B$, can be established from this model. Since both Ax1-asvwords and $A \mathrm{x} B$-asvwords are aligned from the address base of the memory space, any $A \mathrm{x} B$-asvword can be aligned with $B A \mathrm{x} 1$-asvwords as shown in Figure 2.

Figure 2 illustrates the asvword model in which each dash-dotted rectangle/square represents an svword and each red/solid rectangle represents an asv- 
word composed of eight svwords (i.e. $A=8$ ). The two rows show the memory alignment corresponding to the size $b$ of svwords, where $b$ is 1 or 2 (i.e. $B=2$ ), on the same sixteen consecutive memory units with addresses from 0 to 15 . An asvwrite operation can atomically write to some or all of the eight svwords of an asvword. Unlike the aiwrite assignment in the second model, which can atomically write to at most 8 units (or $A$ units), the asvwrite assignment in the third model can atomically write to 16 units (or $A \cdot B$ units) using a single $8 x 2$-asvwrite operation (i.e. write to the whole set of eight 2 -svwords, cf. row $b=2$ ). For an $8 \times 1$-asvword on row $b=1$, there are two methods to update it atomically using the asvwrite operation: i) writing to the whole set of eight 1-svwords using a single 8x1-asvwrite (cf. SIMD core 1) or ii) writing to a subset consisting of four 2-svwords using a single 8x2-asvwrite (cf. SIMD core 2). However, if only one of the eight units of an 8x1-asvword (e.g. unit 14) needs to be updated and the other units (e.g. unit 15) must remain untouched, the only possible method is to write to the unit using a single $8 \mathrm{x} 1$-asvwrite (cf. SIMD core 3 ). The other method, which writes to one 2-svword using a single $8 \times 2$-asvwrite, will have to overwrite another unit that is required to stay untouched (cf. SIMD core 4).

Terminology This paper uses the conventional terminology from bivalency arguments $[8,10,15]$. The configuration of an algorithm at a moment in its execution consists of the state of every shared object and the internal state of every process. A configuration is univalent if all executions continuing from this configuration yield the same consensus value and multivalent otherwise. A configuration is critical if the next operation $o p_{i}$ by any process $p_{i}$ will carry the algorithm from a multivalent to a univalent configuration. The operations $o p_{i}$ are called critical operations. The critical value of a process is the value that would get decided if that process takes the next step after the critical configuration.

\section{Consensus number of the svword model}

Before proving the consensus number of the single-svword assignment, we present the essential features of any wait-free consensus algorithm $\mathcal{A} \mathcal{L} \mathcal{G}$ for $N$ processes using only single- ${ }^{*}$ word assignments and registers, where ${ }^{*}$ word can be svword, aiword or asvword. It has been proven that such an algorithm must have a critical configuration, $C_{0}$, and the next assignment $o p_{i}$ (i.e. the critical operation) by each process $p_{i}$ must write to the same object $\mathcal{O}$ [10]. The object $\mathcal{O}$ consists of memory units.

Lemma 1. The critical assignment op $p_{i}$ by each process $p_{i}$ must atomically write to

- a "single-writer" unit (or 1 W-unit for short) $u_{i}$ written only by $p_{i}$ and

- "two-writer" units (or $2 W$-units for short) $u_{i, j}$ written only by two processes $p_{i}$ and $p_{j}$, where $p_{j}$ 's critical value is different from $p_{i}$ 's, $\forall j \neq i$.

Proof. The proof is similar to the bivalency argument of Theorem 13 in [10]. 


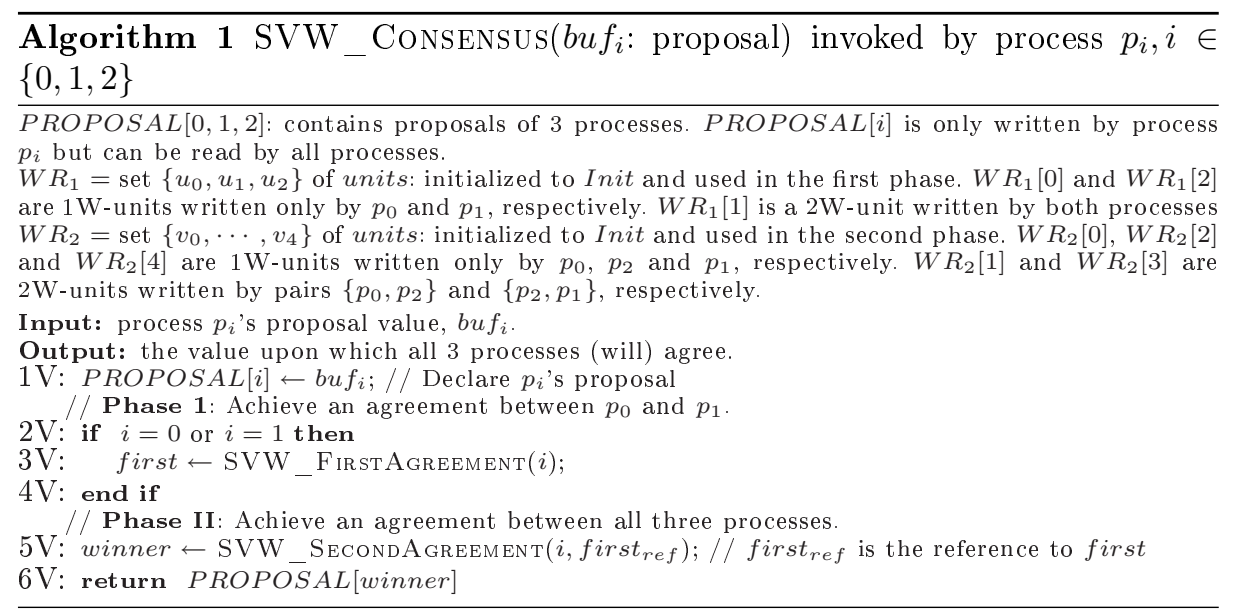

In this section, we first present a wait-free consensus algorithm for 3 processes using only the single-svword assignment with $B \geq 5$ and registers. Then, we prove that we cannot construct any wait-free consensus algorithms for more than 3 processes using only the single-svword assignment and registers regardless of how large $B$ is.

The new wait-free consensus algorithm SVW_CONSENSUS is presented in Algorithm 1. The main idea of the algorithm is to utilize the size-variation feature of the svwrite operation. Since $b$-svwrite can atomically write $b$ values of size 1 unit (instead of just one value of size $b$ units) to $b$ consecutive memory units, keeping the size of values to be atomically written as small as 1 unit will maximize the number of processes for which b-svwrite, together with registers, can solve the consensus problem. Unlike the seminal wait-free consensus algorithm using the $m$-word assignment by Herlihy [10], which requires the word size to be large enough to accommodate a proposal value, the new algorithm stores proposal values in shared memory and uses only two bits (or one unit) to determine the preceding order between two processes. This allows a single-svword assignment to write atomically up to $B$ (or $\frac{B}{2}$ if units are single bits) orderingrelated values. The new algorithm utilizes process unique identifiers, which are an implicit assumption in Herlihy's consensus model [6].

The SVW_Consensus algorithm has two phases. In the first phase, two processes $p_{0}$ and $p_{1}$ will achieve an agreement on their proposal values (cf. Algorithm 2). The agreed value, PROPOSAL[first], is the proposal value of the preceding process, whose SVWRITE (lines $2 \mathrm{SF}$ and $4 \mathrm{SF}$ ) precedes that of the other process (lines 6SF-11SF).

Due to the memory alignment restriction, in order to be able to allocate memory for the $W R_{1}$ variable (cf. Algorithm 1 ) on which $p_{0}$ 's and $p_{1}$ 's SVWRITEs can partly overlap, $p_{0}$ 's and $p_{1}$ 's SVWRITEs are chosen as 2-svwrite and 3-svwrite, respectively. The $W R_{1}$ variable is located in a memory region consisting of 4 consecutive units $\left\{u_{0}, u_{1}, u_{2}, u_{3}\right\}$ of which $u_{0}$ is at an address multiple of 2 and 


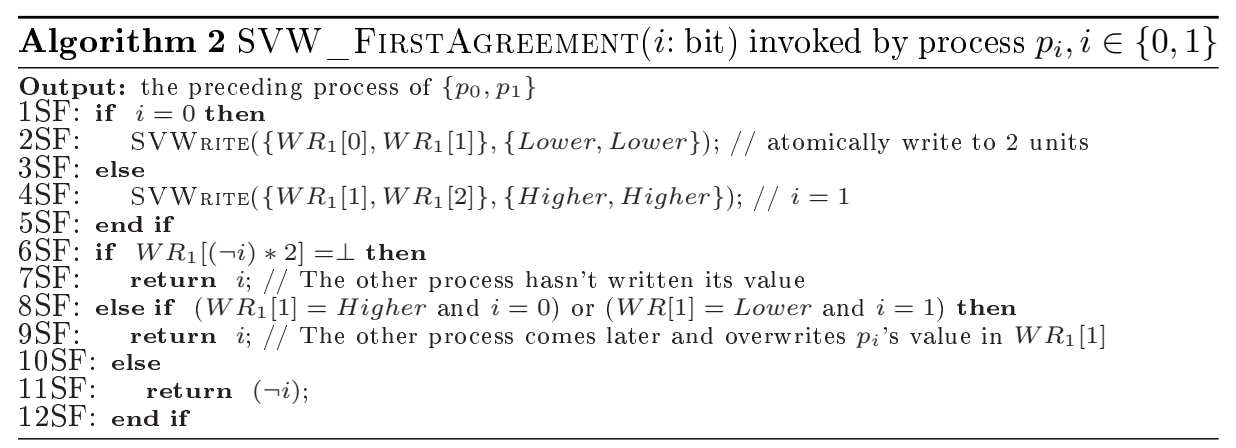

Algorithm 3 SVW_SecondAgreement $\left(i\right.$ : index; first ref $_{\text {: }}$ reference $)$ invoked by process $p_{i}, i \in \bar{\in}\{0,1,2\}$

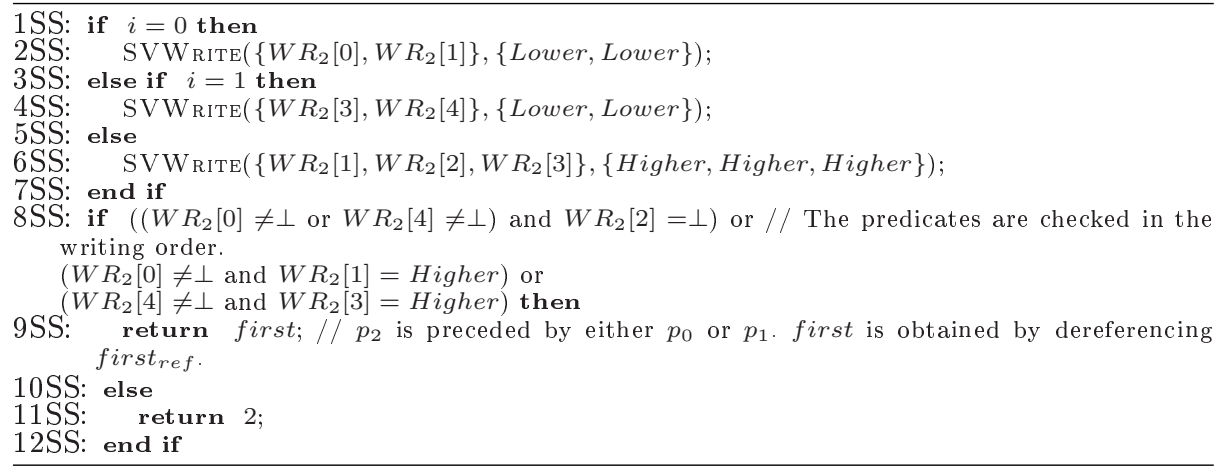

$u_{1}$ at an address multiple of 3 . This memory allocation allows $p_{0}$ and $p_{1}$ to write atomically to the first two units $\left\{u_{0}, u_{1}\right\}$ and the last 3 units $\left\{u_{1}, u_{2}, u_{3}\right\}$, respectively (cf. Figure $3(\mathrm{a})$ ). The $W R_{1}$ variable is the set $\left\{u_{0}, u_{1}, u_{2}\right\}$ (cf. the solid squares in Figure 3(a)), namely $p_{1}$ ignores $u_{3}$ (cf. line 4SF in Algorithm 2).

Subsequently, the agreed value will be used as the critical value of both $p_{0}$ and $p_{1}$ in the second phase in order to achieve an agreement with the other process $p_{2}$ (cf. Algorithm 3 ). Let $p_{\text {first }}$ be the preceding process of $p_{0}$ and $p_{1}$ in the first phase. The second phase returns $p_{\text {first }}$ 's proposal value if either $p_{0}$ or $p_{1}$ precedes $p_{2}$ (line 9SS) and returns $p_{2}$ 's proposal value otherwise.

Units written by processes' SVWRITE are illustrated in Figure 3(b). In order to be able to allocate memory for the $W R_{2}$ variable, process $p_{0}$ 's, $p_{1}$ 's and $p_{2}$ 's SVWRITES are chosen as 2-svwrite, 3-svwrite and 5-svwrite, respectively. The $W R_{2}$ variable is located in a memory region consisting of 7 consecutive units $\left\{u_{0}, \cdots, u_{6}\right\}$ of which $u_{0}$ is at an address multiple of $2, u_{4}$ at an address multiple of 3 and $u_{1}$ at an address multiple of 5 . Since 2, 3 and 5 are prime numbers, we always can find such a memory region. For instance, if the memory address space starts from the unit with index 0 , the memory region from unit 14 to unit 20 can be used for $W R_{2}$ (cf. Figure 1(a)). This memory allocation allows $p_{0}, p_{1}$ and $p_{2}$ to write atomically to the first two units $\left\{u_{0}, u_{1}\right\}$, the last three 


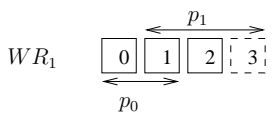

(a) SVW_1stAgreement.

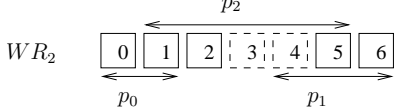

(b) SVW_2ndAgreement.

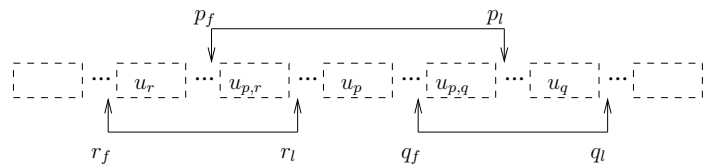

(c) $S=\{p\}, \bar{S}=\{q, r, t\}$

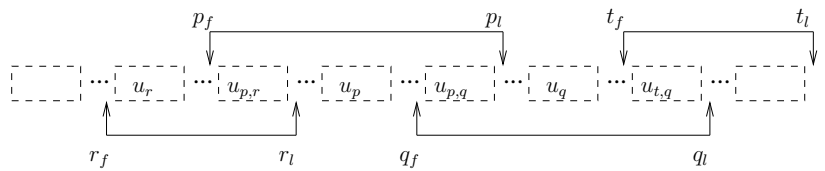

(d) $S=\{p, t\}, \bar{S}=\{q, r\}$

Fig. 3. Illustrations for the SVW_First AGReEMEnT, SVW_SecondAgreEment and Lemma 5.

units $\left\{u_{4}, u_{5}, u_{6}\right\}$ and the five middle units $\left\{u_{1}, \cdots, u_{5}\right\}$, respectively. The $W R_{2}$ variable is the set $\left\{u_{0}, u_{1}, u_{2}, u_{5}, u_{6}\right\}$ (cf. the solid squares in Figure 3(b)).

Lemma 2. The SVW_SECONDAGREEMENT procedure returns index 2 if $p_{2}$ precedes both $p_{0}$ and $p_{1}$. Otherwise, it returns index first.

Lemma 3. The SVW_CONSENSUS algorithm is wait-free and solves the consensus problem for 3 processes.

Proof. It is obvious from the pseudocode in Algorithms 1, 2 and 3 that the SVW_CONSENSUS algorithm is wait-free.

From Lemma 2, the SVW_Consensus algorithm returns the same values for all invoking processes. The value is either PROPOSAL[2] (if $p_{2}$ precedes both $p_{0}$ and $p_{1}$ ) or PROPOSAL[first $]$, first $\in\{0,1\}$ (otherwise).

Lemma 4. The single-svword assignment has consensus number at least 3, $\forall B \geq 5$.

Lemma 5. The single-svword assignment has consensus number at most 3 , $\forall B \geq 2$.

Proof. (Intuition; the full proof is in [9]) We prove the lemma by contradiction. Assume that there is a wait-free consensus algorithm $\mathcal{A L G}$ for four processes $p, q, r$ and $t$. At the critical configuration of the algorithm, we can always divide the set of the four processes into two non-empty subsets $S$ and $\bar{S}$ where $S$ consists of at most two processes with the same critical value called $V$ and $\bar{S}$ consists 
of processes with critical values different from $V$ (If three of the four processes have the same critical value, the other process is chosen as $S$ ). Since the svwrite operation writes to consecutive memory units in the conventional 1-dimensional memory address space, let $\left[k_{f}, k_{l}\right]$ be the range of consecutive units to which a process $k \in\{p, q, r, t\}$ atomically writes using its critical operation $o p_{k}$ (cf. Lemma 1). For any pair of processes $\{h, k\}$, where $h$ and $k$ belong to different subsets $S$ and $\bar{S},\left[h_{f}, h_{l}\right]$ and $\left[k_{f}, k_{l}\right]$ must partly overlap (due to the second requirement of Lemma 1) and none of them are completely covered by ranges $\left[v_{f}, v_{l}\right]$ of the other processes $v$ (due to the first requirements of Lemma 1).

Figures 3(c) and 3(d) illustrate the proof when $S$ consists of one and two processes, respectively. In Figure $3(\mathrm{c})$, the range $\left[t_{f}, t_{l}\right]$ of process $t$ cannot partly overlap with that of process $p$ without completely covering (or being covered by) the range of process $r$ or $q$. In Figure 3(d), $t$ and $r$ belong to different subsets $S$ and $\bar{S}$, respectively, but their ranges cannot partly overlap.

Theorem 1. The single-svword assignment has consensus number 3 when $B \geq$ 5 and three is the upper bound of consensus numbers of single-svword assignments $\forall B \geq 2$.

\section{Consensus number of the aiword model}

In this section, we prove that the single-aiword assignment (or aiwrite for short) has consensus number exactly $\left\lfloor\frac{A+1}{2}\right\rfloor$. First, we prove that the aiwrite operation has consensus number at least $\left\lfloor\frac{A+1}{2}\right\rfloor$. We prove this by presenting a wait-free consensus algorithm AIW_CONSENSUS for $N=\left\lfloor\frac{A+1}{2}\right\rfloor$ processes (cf. Algorithm 4) using only the aiwrite operation and registers. Subsequently, we prove that there is no wait-free consensus algorithm for $N+1$ processes using only the aiwrite operation and registers.

The main idea of the AIW_Consensus algorithm is to gradually extend the set $S$ of processes agreeing on the same value by one at a time. This is to minimize the number of $1 \mathrm{~W}$ - and $2 \mathrm{~W}$-units that must be written atomically by the aiword operation (cf. Lemma 9). The algorithm consists of $N$ rounds and a process $p_{i}, i \in[1, N]$, participates from round $r_{i}$ to round $r_{N}$. A process $p_{i}$ leaves a round $r_{j}, j \geq i$, and enters the next round $r_{j+1}$ when it reads the value upon which all processes in the round $r_{j}$ (will) agree. A round $r_{j}$ starts with the first process that enters the round, and ends when all $j$ processes $p_{i}, 1 \leq i \leq j$, have left the round. At the end of a round $r_{j}$, the set $S$ consists of $j$ processes $p_{i}, 1 \leq i \leq j$.

Lemma 6. All correct processes ${ }^{6} p_{i}$ agree on the same value in round $r_{j}$, where $1 \leq i \leq j \leq N$.

With the assumption that AIWRITE can atomically write to $p_{j}$ 's units at line $2 \mathrm{I}$ and $p_{i}$ 's units at line 11I, it follows directly from Lemma 6 that all the $N$ processes will achieve an agreement in round $r_{N}$.

\footnotetext{
${ }^{6}$ A correct process is a process that does not crash.
} 


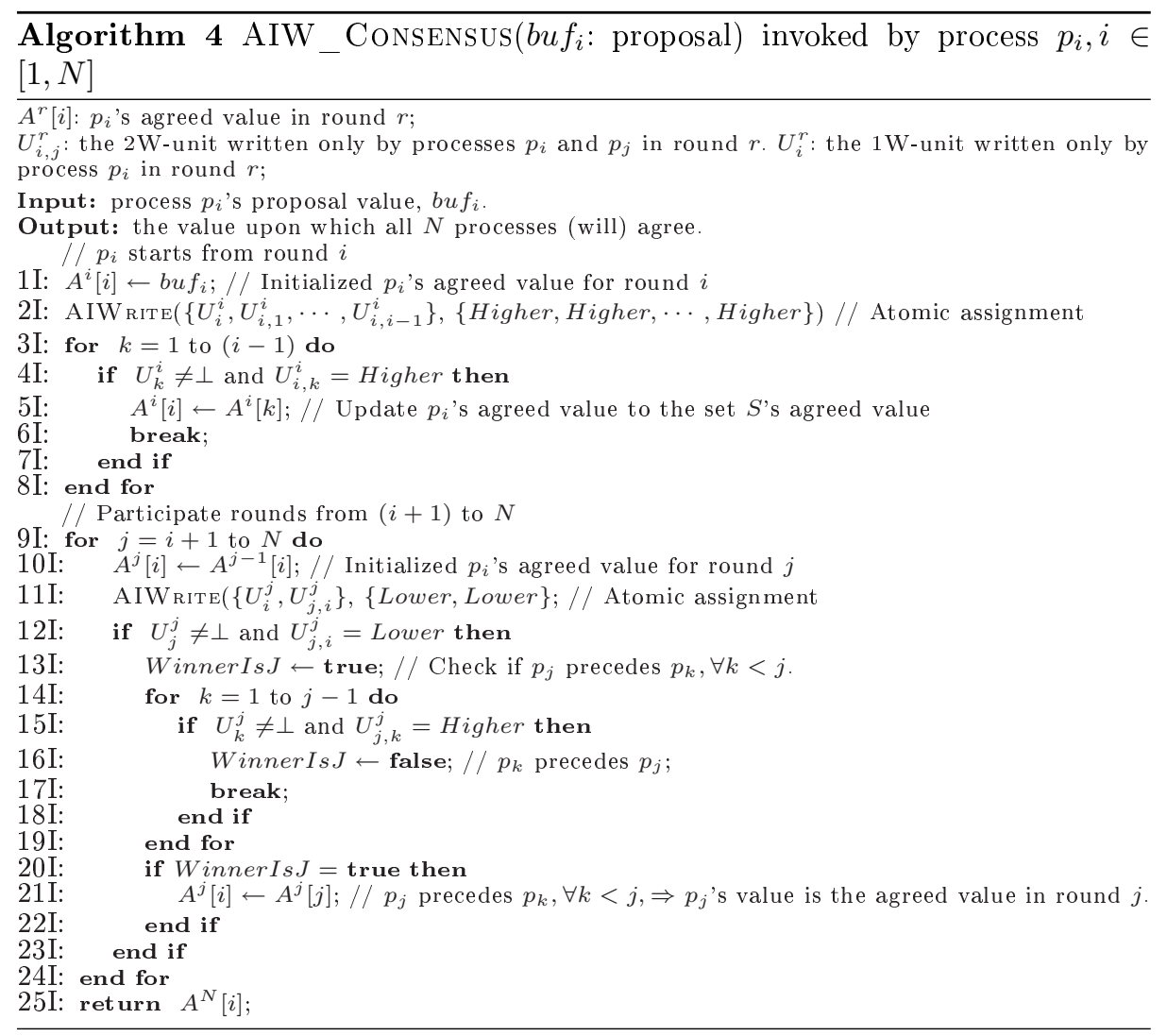

Lemma 7. The AIW_CONSENSUS algorithm is wait-free and can solve the consensus problem for $N=\left\lfloor\frac{A+1}{2}\right\rfloor$ processes.

Proof. (Intuition; the full proof is in [9]) The time complexity for a process using AIW_Consensus to achieve an agreement among $N$ processes is $O\left(N^{2}\right)$ due to the for-loops at lines 9I and 14I. Therefore, the AIW_Consensus algorithm is wait-free.

From Lemma 6, the AIW_Consensus algorithm can solve the consensus problem for $N=\left\lfloor\frac{A+1}{2}\right\rfloor$ processes if AIWRITE can atomically write to $p_{j}$ 's units at line $2 \mathrm{I}$ and $p_{i}$ 's units at line 11I. Indeed, since $N=\left\lfloor\frac{A+1}{2}\right\rfloor$, an $A$-unit aiword (or A-aiword for short) can accommodate both $(N-1) 2 \mathrm{~W}$-units $U_{N, i}^{N}, 1 \leq i<N$, and $N 1 \mathrm{~W}$-units $U_{k}^{N}, 1 \leq k \leq N$, used in round $r_{N}$. Since the single-aiword assignment AIWriTe can atomically write to an arbitrary subset of the $A$ units of an aiword and leave the other units untouched, each process $p_{k}, 1 \leq k \leq N$ can atomically write to only ${ }^{7}$ its $1 \mathrm{~W}$ and $2 \mathrm{~W}$ units.

Lemma 8. The single-aiword assignment has consensus number at least $\left\lfloor\frac{A+1}{2}\right\rfloor$.

7 "Only" here means to leave other units untouched. 


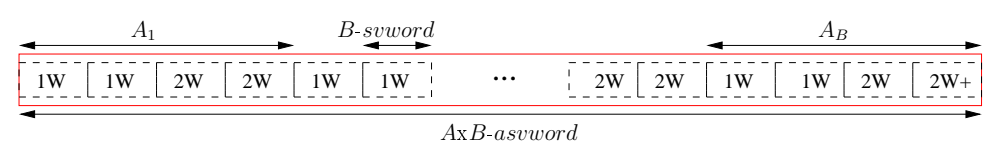

Fig. 4. An illustration for grouping units in the asvword model.

Lemma 9. The single-aiword assignment has consensus number at most $\left\lfloor\frac{A+1}{2}\right\rfloor$.

Proof. (Intuition; the full proof is in [9]) We prove this lemma by contradiction. Assume that there is a wait-free consensus algorithm $\mathcal{A L G}$ for $N$ processes where $N \geq\left\lfloor\frac{A+1}{2}\right\rfloor+1$. At the critical configuration of the $\mathcal{A L G}$ algorithm, we divide $N$ processes into two subsets $S$ and $\bar{S}$ where $S$ consists of processes with the same critical value called $V$ and $\bar{S}$ consists of processes with critical values different from $V$. Let $|S|$ and $|\bar{S}|$ to be the sizes of the subsets, we have $|S|+|\bar{S}|=N$. Due to the memory alignment restriction, all the $1 \mathrm{~W}$-units and $2 \mathrm{~W}$-units used by critical assignments in the $\mathcal{A L G}$ algorithm must be located in the same $A$-aiword called $A I$. Let $M$ be the number of the $1 \mathrm{~W}-/ 2 \mathrm{~W}$-units, we have $M \leq A$.

Since $\mathcal{A L G}$ is a wait-free consensus algorithm for $N$ processes, it follows from Lemma 1 that there are $N 1 \mathrm{~W}$-units and $|S| \cdot|\bar{S}| 2 \mathrm{~W}$-units, i.e. $M=N+|S| \cdot|\bar{S}|$. Since $1 \leq|S| \leq(N-1), M \geq(2 N-1)$. Since $N \geq\left\lfloor\frac{A+1}{2}\right\rfloor+1$ due to the hypothesis, $M \geq(A+1)$ must hold. This contradicts the requirement $M \leq A$.

Theorem 2. The single-aiword assignment has consensus number exactly $\left\lfloor\frac{A+1}{2}\right\rfloor$.

\section{Consensus number of the asvword model}

The intuition behind the higher consensus number of the asvword model compared with the aiword model (cf. Equation (1)) is that process $p_{N}$ in Algorithm 4 can atomically write to $A \cdot B$ units using $A x B$-asvwrite instead of only $A$ units using $A$-aiwrite. To prevent $p_{N}$ from overwriting unintended units (as illustrated by SIMD core 4 in Figure 2), each $B$-svword located in $A_{l}, 1 \leq l \leq B$, contains either $1 \mathrm{~W}$-units or $2 \mathrm{~W}$-units but not both as illustrated in Figure 4 , where $B$ svwords labeled " $1 \mathrm{~W}$ " contain only $1 \mathrm{~W}$-units and $B$-svwords labeled " $2 \mathrm{~W}$ " contain only $2 \mathrm{~W}$-units. This allows $p_{N}$ to atomically write to only $B$-svwords with $2 \mathrm{~W}$ units $U_{N, i}^{N}$ (and keep 1W-unit $U_{i}^{N}, i \neq N$, untouched) using $A x B$-asvwrite. For each process $p_{i}, i \neq N$, its $1 \mathrm{~W}$-unit $U_{i}^{N}$ and $2 \mathrm{~W}$-unit $U_{N, i}^{N}$ are located in two $B$-svwords labeled "1W" and "2W", respectively, that belong to the same $A_{l}$. This allows $p_{i}$ to atomically write to only its two units using Ax1-asvwrite. A complete proof of the exact consensus number can be found in the full version of this paper [9].

Acknowledgements The authors wish to thank the anonymous reviewers for their helpful and thorough comments on the earlier version of this paper. Phuong 
Ha's and Otto Anshus's work was supported by the Norwegian Research Council (grant numbers 159936/V30 and 155550/420). Philippas Tsigas's work was supported by the Swedish Research Council (VR) (grant number 37252706).

\section{References}

1. Cell Broadband Engine Architecture, version 1.01. IBM, Sony and Toshiba Corporations, 2006.

2. NVIDIA CUDA Compute Unified Device Architecture, Programming Guide, version 1.1. NVIDIA Corporation, 2007.

3. S. V. Adve and K. Gharachorloo. Shared memory consistency models: A tutorial. Computer, 29(12):66-76, 1996.

4. Y. Afek, M. Merritt, and G. Taubenfeld. The power of multi-objects (extended abstract). In PODC '96: Proceedings of the fifteenth annual ACM symposium on Principles of distributed computing, pages 213-222, 1996.

5. H. Attiya and J. Welch. Distributed Computing: Fundamentals, Simulations, and Advanced Topics. John Wiley and Sons, Inc., 2004.

6. H. Buhrman, A. Panconesi, R. Silvestri, and P. Vitanyi. On the importance of having an identity or, is consensus really universal? Distrib. Comput., 18(3):167$176,2006$.

7. I. Castano and P. Micikevicius. Personal communication. NVIDIA, 2008.

8. M. J. Fischer, N. A. Lynch, and M. S. Paterson. Impossibility of distributed consensus with one faulty process. J. ACM, 32(2):374-382, 1985.

9. P. H. Ha, P. Tsigas, and O. J. Anshus. The synchronization power of coalesced memory accesses. Technical report CS:2008-68, University of Tromsø, Norway, 2008.

10. M. Herlihy. Wait-free synchronization. ACM Transaction on Programming and Systems, 11(1):124-149, Jan. 1991.

11. P. Jayanti and S. Khanna. On the power of multi-objects. In WDAG '97: Proceedings of the 11th International Workshop on Distributed Algorithms, pages 320-332, 1997.

12. L. Lamport. How to make a multiprocessor computer that correctly executes multiprocess progranm. IEEE Trans. Comput., 28(9):690-691, 1979.

13. J. D. Owens, D. Luebke, N. Govindaraju, M. Harris, J. Krüger, A. E. Lefohn, and T. J. Purcell. A survey of general-purpose computation on graphics hardware. Computer Graphics Forum, 26(1):80-113, 2007.

14. S. Ramamurthy, M. Moir, and J. H. Anderson. Real-time object sharing with minimal system support. In Proc. of Symp. on Principles of Distributed Computing $(P O D C)$, pages 233-242, 1996.

15. E. Ruppert. Determining consensus numbers. In Proc. of Symp. on Principles of Distributed Computing (PODC), pages 93-99, 1997.

16. E. Ruppert. Consensus numbers of multi-objects. In Proc. of Symp. on Principles of Distributed Computing (PODC), pages 211-217, 1998. 